Gdańsk 2019, Nr. 40

https://doi.org/10.26881/sgg.2019.40.14

\title{
Caroline Scholzen
}

Universität Salzburg / Uniwersytet w Salzburgu

https://orcid.org/0000-0002-8279-1445

\author{
Doppelgänger - an Ketten gelegt: Zum Einfluss \\ von Emanuel Swedenborg und Salomon Maimon \\ auf E. T. A. Hoffmann und Franz Kafka
}

\begin{abstract}
In Träume eines Geistersehers, erläutert durch Träume der Metaphysik parallelisiert Kant nicht nur die Leibniz-Wolffsche-Philosophie mit der spiritistischen Theosophie Swedenborgs, sondern versucht vor allem die rationalistische Philosophie vor einer Geisterseherei zu bewahren. Dass damit für den „Bürger zweier Welten“ die Vermischung von durchdringlichen und undurchdringlichen Substanzen nicht gebannt ist, sondern vielmehr zum unheimlichen Doppelgängertum mutiert, lässt sich durch die Werke des Kant- und Swedenborg-Lesers Hoffmann aufweisen. Wie es darüber hinaus Kafka vermag, die „Doppelbürgerschaft“ als semantische wie performative Kampfbewegung zu realisieren, wird ein weiterer Untersuchungsgegenstand sein.
\end{abstract}

Schlüsselwörter: Immanuel Kant, „Bürger zweier Welten“, Geistersehen, Doppelgänger

Doppelgänger - enchained: the influence of Emanuel Swedenborg and Salomon Maimon on E. T. A. Hoffmann and Franz Kafka. In Dreams of a Spirit-Seer, illustrated by Dreams of Metaphysics, Immanuel Kant relates the philosophies of Gottfried Wilhelm Leibniz and Christian Wolff to the spiritistic theosophy of Emanuel Swedenborg to prevent rationalism from changing into ghost-seeing. As human beings are "citizens of two worlds", the danger of confounding permeable and impermeable substances is not averted. The consequence is rather that eerie lookalikes emerge, which I will show using the example of E. T. A. Hoffmann, who read both Kant and Swedenborg. My paper also examines Franz Kafka's successful realizing of the "double citizenship" as a semantic and performative combat move.

Keywords: Immanuel Kant, “citizen of two worlds”, ghost seeing, doppelganger

Im Jahr 1804 fertigte E. T. A. Hoffmann eine Federzeichnung an, auf der sein Kopf hinter demjenigen seines Freundes Theodor Gottlieb Hippel dargestellt ist. Beide Köpfe sind in eine Richtung gewendet und mit der Unterschrift „Castor und Pollux“ versehen (Steinecke 1997: 18). Dass dieses antike Dioskuren-Zwillingspaar aus einem unsterblichen und einem sterblichen Part besteht, wird eine nicht unwesentliche Rolle für die folgenden Ausführungen 
zu Doppelgängern oder Doppelgängerschaft im Werk von E. T. A. Hoffmann und Franz Kafka spielen.

Die Freundschaft zu Hippel stilisiert Hoffmann gerne als Doppelgängertum eines schönen und vernünftigen Freundes - Hippel - mit einem wankelmütigen, künstlerischen, düster-hässlichen Gegenüber - Hoffmann. Nicht nur über sein Rechtsstudium in Königsberg, sondern vor allem über die intensive Freundschaft zu Hippel, der gleichzeitig ein Freund Immanuel Kants war, kann Hoffmanns genaue Kenntnis von Kants Werk angenommen werden. Dass er auch Kants Schrift Träume eines Geistersehers, erläutert durch Träume der Metaphysik gekannt haben muss, geht aus seiner Bewunderung für Friedrich Schillers Werk Der Geisterseher. Aus den Memoiren des Grafen von $O$ hervor, welches eine literarische Auseinandersetzung mit Kants, vorkritischer 'Schrift darstellt. Besonders für Der Sandmann kommen laut Claudia Liebrand die genannten Werke Schillers und Kants „als Anspielungsfolie in Betracht" (2009: 178). Kant schließt in Träume eines Geistersehers seine Kritik an den traditionellen Metaphysikern und dem zu seiner Zeit in Stockholm lebenden Wissenschaftler und Mystiker Emanuel Swedenborg insofern kurz, als er beide - die einen als „Träumer der Vernunft“, die anderen als „Träumer der Empfindung“ (TG: 342$)^{1}$ - für ihre Schwärmerei und deren immanente Tendenz zur Privatisierung und Elitisierung kritisiert. Da sowohl Kant als auch Swedenborg von der Metaphysik Gottfried Wilhelm Leibniz und Christian Wolffs beeinflusst sind, versucht sich Kant vehement von einer Ontologisierung der Metaphysik abzugrenzen. Swedenborg wird dennoch oft als Kants „Zwillingsbruder" bezeichnet, von dem letzterer sich - so das Gerücht - auch durch die Änderung seines ursprünglichen Taufnamens Emanuel zu Immanuel zu unterscheiden versuchte. Kant stört nicht Swedenborgs Behauptung, dass es Geister gibt, setzt er doch selbst voraus, dass der Mensch zur intelligiblen Welt gehört, ihn ärgert vielmehr Swedenborgs Behauptung, einen exklusiven Umgang mit Geistern zu pflegen und mit ihnen in einer äußeren, sinnlich wahrnehmbaren Kommunikation zu stehen. Kant wendet sich laut Friedrich Balke gegen Swedenborgs „Diskursstrategie“ (2005: 303-305), die auf die Privatisierung von subjektiven Erfahrungen einer intelligiblen Kommunikation abzielt. Aus dem Anspruch einer bürgerlichen Gesellschaft heraus, in der jede und jeder die gleichen Erfahrungen und allgemeinen Erkenntnisse machen kann, verachtet Kant Swedenborgs private Geisterseherei. Dass Swedenborgs Schriften durchaus beliebt waren und in der zeitgenössischen Gesellschaft viele Gerüchte über seine Geistererlebnisse kursierten, hält Kant für die Folgen eines das Projekt der Aufklärung gefährdenden Halb- und Nicht-Wissens (Kohns 2007: 123).

Kants Reaktion auf Swedenborg und die um seine Geistererfahrungen kreisenden Gerüchte ist vergleichbar mit vielen in Hoffmanns Werk beschriebenen Konflikten. Immer wieder werden Protagonisten, die von ihren und nur ihren Einbildungen und Erfahrungen berichten, als Geisterseher beschimpft. In Der Magnetiseur wird dieses Geistersehen sogar

1 Immanuel Kants Werke werden im Folgenden mit den Siglen TG (= Träume eines Geistersehers, erläutert durch Träume der Metaphysik), Anth, (=Anthropologie in pragmatischer Hinsicht) nach folgender Ausgabe zitiert: Immanuel Kant: Gesammelte Schriften. Abhandlungen nach 1781. Hg. von der Preussischen Akademie der Wissenschaften u. a. Berlin 1900ff. (AA). 
direkt auf Emanuel Swedenborg bezogen. So wird der Protagonist Alban von dem Maler Bickert folgendermaßen attackiert:

Da haben wir den Wunder-Doktor! rief Bickert, als man die schlafende Marie in ihr Zimmer gebracht [...] hatte. - Der tiefsinnige Blick des Geistersehers - das feierliche Wesen - das prophetische Voraussagen - das Fläschchen mit dem Wunderelixier. - Ich habe nur gepaßt, ob er nicht, wie Schwedenborg vor unsern Augen in der Luft verdampfen [...] würde. (II/1: 202)

Oft stellt Hoffmann seine Geister sehenden Protagonisten in ihrer Abhängigkeit von gesellschaftlichem Klatsch und Gerüchten dar. So ist beispielsweise die Hauptfigur Theodor in Das öde Haus auf das Halbwissen anderer über genau dasjenige Haus angewiesen, welches er nicht aufhören kann von der Straße über einen Spiegel aus anzustarren (III: 166-169). Außerdem bezeichnen sich viele Protagonisten in Auseinandersetzung mit ihrem jeweiligen „,verständigen“" (III: 29) und „magistermäßig[en]“ (III: 24) Gegenüber als „törichte“ (II/2:71; IV: 792), „träumerische“ (III: 173), „überspannte“ (III: 168) und in „verwunderliche Gedanken verstrickte“ Geisterseher (III: 166-167), als „abgeschmackte“ Geisterseher (II/2: 71; III: 37) oder wie Nathanael in seinem ersten Brief an Lothar zu Anfang von Der Sandmann als „aberwitziger" Geisterseher (III: 11).

Hoffmann war nicht nur mit Kants Werk vertraut, sondern auch die Schriften Swedenborgs waren ihm über die Vorträge und das Werk des von ihm sehr geschätzten Gotthilf Heinrich von Schubert bekannt. Daher muss festgehalten werden, dass er die Gefahren eines Sprechens von privaten, subjektiven Erfahrungen ebenso reflektiert wie die Folgen einer Rede, die ausschließlich von allgemeinen Erkenntnissen und deren verständigem Nachvollzug handelt. Durch die Ironisierung beider Positionen nimmt Hoffmann eine Doppelposition ein, die die persönliche wie gesellschaftliche Gefahr von privater Rede und einem vermeintlich „reinen“" „kritischen“ Sprechen bloßstellt. Hoffmann weist in seinem Werk immer wieder auf, dass sowohl eine allein verstandeslogisch-schematisierte als auch eine lediglich assoziativ-imaginäre Erfassung den Menschen nicht darzustellen vermag. Besonders deutlich wird dies an der Figur Clara aus Der Sandmann: Clara wird mit einer doppelt gerichteten Rede konfrontiert, auf die weder sie noch Nathanael in einer entsprechend ambivalenten Weise reagieren können. Sie erreicht ein Brief von Nathanael, der zwar an sie adressiert, aber nicht an sie gerichtet ist. Daher bricht sie das Briefgeheimnis noch nicht mit dem Öffnen, jedoch mit dem darauffolgenden Lesen des an ihren Bruder gerichteten Schreibens. Das Briefgeheimnis bezieht sich auf die Nachricht zwischen zwei Menschen, die sich in ihrer jeweiligen Existenz gedanklich voraussetzen, ohne die sinnlich-körperliche Präsenz des anderen wahrnehmen zu können. Adressant und Adressat werden vom Briefgeheimnis als abwesend Anwesende geschützt. Mit dem Lesen des Briefes verletzt Klara dieses Briefgeheimnis und damit das Grundrecht ihres Verlobten und ihres Bruders auf private Kommunikation. Gleichzeitig erzwingt sie die Partizipation an der intelligiblen Kommunikationsgemeinschaft, die zwischen Nathanael und Lothar besteht. Die „verständige“ Clara verhält sich damit ebenso unklar wie der Hauptvertreter der

2 Hoffmann, E. T. A.: Sämtliche Werke in 6 Bänden (1985-2004). Hgg. von H Steinecke u. W. Segebrecht, Frankfurt a. M.: Bibliothek deutscher Klassiker. Diese Ausgabe wird im laufenden Text mit der Angabe des Bandes, der Abteilung und der Seitennummer, ohne Sigle, zitiert. 
Aufklärung selbst: Sie akzeptiert genauso wenig wie Kant die Existenz und das Reden von privaten Geistern, setzt jedoch gleichzeitig eine fraglose Partizipation an einer intelligiblen Geisterwelt voraus. Mit der Doppelgerichtetheit ${ }^{3}$ von Sprache, die letztlich ein Resultat der Doppelgesichtigkeit der Einbildungskraft (Kohns 2007: 177) ist, kann sie ebenso wenig umgehen wie Kant. Oliver Kohns hält zur Doppelfunktion der assoziativen Einbildungskraft fest:

Einerseits ist die Assoziation das grundlegende Verfahren des Verstandes, indem sie Vorstellungen (ideas) aneinander bindet und so für Bewusstseinsakte (Erinnern oder Urteilen) überhaupt erst zugänglich macht; andererseits kann sie diese Leistung nur dadurch vollbringen, dass sie dem Verstand abwesende Vorstellungen als anwesend re-präsentiert und ihn so dazu verleitet, potentiell den Kontakt zur ,Wirklichkeit' zu verlieren und sich in einer Welt eingebildeter Chimären zu verirren (2007: 138; Herv. i. Orig.).

Gegen Kants frühe Versuche, dichterisches, „phantastisches“ Sprechen aus kritischem Denken zu vertreiben, legt Hoffmanns Literatur bloß, dass jegliche intelligible Ab- und Versicherung auf der vermeintlichen „Krankheit“ gründet, das Zeichen mit dem Bezeichneten zu verwechseln. Auch wenn sich Clara eine rationale Immunität gegenüber Geistererscheinungen zugutehält, so unterliegt sie nicht minder dieser Verwechslung: Am Ende ihres eigenen Briefes an Nathanael widerspricht sie erheblich ihrem aufgeklärten Standpunkt, wenn sie schreibt:

Ich habe mir vorgenommen, bei Dir zu erscheinen, wie Dein Schutzgeist, und den häßlichen Coppola, sollte er es sich etwa beikommen lassen, Dir im Traum beschwerlich zu fallen, mit lautem Lachen fortzubannen. Ganz und gar nicht fürchte ich mich vor ihm und vor seinen garstigen Fäusten, er soll mir weder als Advokat eine Näscherei, noch als Sandmann die Augen verderben. (III: 23)

Sie inszeniert sich hier in ihrer Intelligibilität - als anwesend abwesender Geist - und damit gerade in jenem Zustand, den sie nicht nur mit dem Lesen des Briefes nicht würdigte, sondern in Bezug auf Nathanaels Erfahrungen auch belächelt. Clara entspricht hier sowohl Nathanaels ständiger Vertauschung von Literalem und Figuralem (Liebrand 1996: 88) als auch der von ihm für sie verwendeten Bezeichnung - sie realisiert sich als sein „Engelsbild“ (III: 25). Dadurch, dass Clara jedoch Nathanaels dichterischer Sprache keinen Respekt zollt und auch die Bildlichkeit ihrer eigenen Sprache auf semantischer Ebene abzuwehren versucht, kann und muss der einem „tropische[n] Prinzip“ (Liebrand 1996: 88) folgende Nathanael Claras unheimlich-heimlichen, da nichtrationalen Sprachrest außerhalb ihrer Sprache suchen. Bald lässt er daher einen Brief an Clara liegen, um nicht länger in einem intelligiblen, sondern konkreten Gegenüber Claras ein- und d. h. in ihrer Sprache ausgeschlossene Doppelgängerin Olympia zu beobachten. (Das Nathanaels Fenster gegenüberliegende Haus, in dem Olympia eingeschlossen ist, kann daher als Realisierung einer Haus-Metapher verstanden werden, die nicht nur psychoanalytisch, sondern vor allem im Sinne einer antiken ars memoriae näher zu untersuchen wäre.)

3 Eine doppelgerichtete Rede ist laut Michail Bachtin Folge der Tatsache, dass ein Äußerungs- bzw. Handlungssubjekt in seinem ,verantwortlichen Handlungs-Vollzug“ immer als gleichzeitig wahrnehmendes Subjekt wie wahrgenommenes Objekt verstanden werden muss (Bachtin 2011: 34, 64). Sie ist die Grundlage für die von Bachtin später entwickelte Theorie der Dialogizität. 
Während Hoffmann auf die implizite Gefahr des metaphorischen Sprechens gerade durch dieses zu verweisen versucht, warnt Kant lediglich davor, die symbolischen Verknüpfungen wörtlich zu nehmen und die Phantasie „dem innern Zustande der Seele gemäß“ (TG: 340) so zu verstärken, dass diese als äußere Erscheinungen wahrgenommen werden. Da jemand, der sich symbolisch oder metaphorisch ausdrückt, „noch wenig Begriffe des Verstandes“ (Anth: 191) habe, lehre das Projekt der Aufklärung die „einige Zeit hindurch nützliche und nöthige Hülle von der Sache selbst zu unterscheiden“" (Anth: 192). Hoffmanns Literatur weist jedoch auf, dass diese Unterscheidung hinfällig ist, sobald die Funktion von Medien mitreflektiert wird. Diese sind für ihn nicht länger einfache Vermittlungsträger, sondern beanspruchen den Status von anwesend Abwesenden. Diesem zu schützenden und leicht zu schädigenden Anspruch kann sich Clara nicht entziehen, auch wenn die Gerichtetheit des von ihr empfangenen Briefes „verständig“ zwischen Hülle und Sache zu unterscheiden scheint. Während Kant gegen Swedenborgs Geistersehungen anführt, dass eine immaterielle Substanz aufgrund ihrer Durchdringlichkeit einer materiellen nicht „im Wege liegen“( TG: 327) könne, sind sich der Kant-Leser Friedrich Schiller (Weissberg 1990: 125-160) und der Schiller-Leser E. T. A. Hoffmann anderer Möglichkeiten eines anderen Sachverhalts bewusst: Sie reflektieren, dass es durch das schriftliche Medium einem durchdringenden Geist sehr wohl möglich ist, nicht nur mit einer menschlichen Person in einem Raum zu sein, sondern durch einen intelligiblen Anspruch auch dieser „im Wege“ zu liegen. Hoffmann problematisiert geradezu die Möglichkeit von durch Schrift erzeugten „optischen Täuschungen“ und setzt sie nicht, wie Friedrich Kittler (1994: 229) annimmt, als „gelungen“ voraus. Gerade am Vorwort von Die Elixiere des Teufels kann man dies erkennen, welches folgendermaßen beginnt:

Gern möchte ich dich, günstiger Leser! unter jene dunkle Platanen führen, wo ich die seltsame Geschichte des Bruders Medardus zum erstenmale las. Du würdest dich mit mir auf dieselbe [...] steinerne Bank setzen; du würdest, so wie ich, recht sehnsüchtig nach den blauen Bergen schauen [...]. Aber nun wendest du dich um, und erblickest kaum zwanzig Schritte hinter uns ein gotisches Gebäude, dessen Portal reich mit Statüen verziert ist. - Durch die dunklen Zweige der Platanen schauen dich Heiligenbilder recht mit klaren, lebendigen Augen an; es sind die frischen Freskogemälde, die auf der breiten Mauer prangen. [...] Sind denn die Heiligenbilder lebendig worden und herabgestiegen von den hohen Simsen? - Dich umwehen die geheimnisvollen Schauer der wunderbaren Sagen und Legenden die dort abgebildet, dir ist, als geschähe Alles vor deinen Augen, und willig magst du daran glauben. In dieser Stimmung liesest du die Geschichte des Medardus, und wohl magst du auch dann die sonderbaren Visionen des Mönchs für mehr halten als für das regellose Spiel der erhitzten Einbildungskraft. (II/2: 11)

Der mit dem Leser vollzogene textuelle Doppel-(Ein)gang wird hier gerade nicht als gelungen markiert. Sitzen der sich als Herausgeber bezeichnende Erzähler und der Leser zwar auf „derselben Bank“ und sind sie sogar beide Leser der „seltsamen Geschichte“, so liegt doch der eine Lesevorgang weit vor dem anderen. Die zwei Leser schauen wie Hippel und Hoffmann auf der erwähnten Federzeichnung zwar zunächst in die gleiche Richtung, aber der den Erzähler in seinem Lesen doppelnde Leser wendet sich alsbald vom Erzähler ab und den „Heiligenbildern“ zu. Der Leser wird als williger Gläubiger bezeichnet und damit Lesen per se als Geistersehen markiert. Immer wieder stellt Hoffmann die Schrift als gefährliches Manipulationsmedium dar, welches „sköne Oke“ (III: 35) zugleich voraussetzt wie produziert. „Doppelgesichtigkeit“ (Kohns 2007: 177) durch Einbildung betrifft bei ihm 
alle am Äußerungsakt beteiligten Subjekte - Erzähler, Leser und Figuren. Während sich diese andauernd - vor allem in Das öde Haus oder in Die Serapionsbrüder - „Denkt euch“ zurufen, bevor sie eine Erzählung beginnen, vermag die Einbildungskraft mitunter auch auf die Erzählinstanz zu wirken. So unterbricht beispielsweise der Erzähler von Der Sandmann seine Erzählung, weil er „nicht wegschauen kann“, als in einem „Augenblick“ Claras Bild ihm „lebendig vor Augen“ steht (III: 27). Wichtig bleibt dabei jedoch, dass diese Unterbrechung lediglich auf semantischer, nicht auf performativer Ebene stattfindet. Er lässt sein Erzählen im Gegensatz zu Nathanael nicht unter- oder aufbrechen. Während Nathanael den Brief an Clara durch den Blick auf Olympia liegenlässt, setzt der Erzähler den Brief an seinen Leser unbeirrt fort. ${ }^{4}$

Hoffmann nimmt die kantische Kritik am metaphorischen Sprachgebrauch sehr ernst und hält gleichzeitig wenig von Kants Vorhaben, die Traumgestalten der Sprache mit Hilfe einer nach dem Vorbild der Geometrie gebildeten „reinen“ Sprache bändigen zu wollen. Es erscheint daher wie eine Kant-Replik, wenn Hoffmann am Ende des Vorworts der Elixiere genau den für „verloren“ hält, „der mit jener Erkenntnis die Kraft gewonnen glaubt, jenen Faden gewaltsam zu zerreißen, und es aufzunehmen mit der dunklen Macht, die über uns gebietet“ (II/2: 12).

Der Faden der Einbildungskraft, der aus vergesellschaftenden Begriffe besteht - was eine von Kant unternommene Eindeutschung des Begriffs „Assoziation“ darstellt (Kohns 2007: 134) -, und den Hoffmann vor seinem kritischen Zerreißen schützen möchte, lässt sich auf die Kette zurückführen, von der Leibniz in Von dem Verhängnisse geschrieben hat:

Denn das Verhängniß besteht darin, daß alles an einander hänget wie eine Kette [...]. Die alten Poeten, als Homerus und andere, haben es die güldene Kette genennet, so Jupiter vom Himmel herab hängen lasse, so sich nicht zerreißen lässet, man hänge daran, was man wolle. Und diese Kette besteht in den Verfolg der Ursachen und der Wirkungen. (Leibniz 1996: 337)

Die Ketten-Metaphorik verwendet Leibniz hier, um das Prinzip des zureichenden Grundes und das aus diesem folgende der Kontinuität zu veranschaulichen. Hoffmann begreift das Prinzip des zureichenden Grundes im Sinne Leibniz als Versicherung der Realmöglichkeit einer jeden sinnlich-materiellen Kreatur - vor allem die des menschlichen Individuums. ${ }^{5}$ Kant hingegen versucht, die leibnizsche „Kette“ oder „Himmelsleiter“ mit der Newtonschen Theorie der Wechselkräfte von Attraktion und Repulsion zu unterbrechen. In Versuch den Begriff der negativen Größen in die Weltweisheit einzuführen bezieht er das mathematische Konzept von -1 und +1 sowie das kinematische Kräfteparallelogramm auf die Monade und gibt damit ihren „idealistischen Gehalt" auf (Kaulbach 1960:71). Hintergrund von Kants Kritik an Leibniz“ Prinzip des zureichenden Grundes ist wie in Träume eines Geistersehers die Voraussetzung der substantiellen, physischen Ausdehnung (Kaulbach 1960: 70-74, 90-95). Man könnte

4 Claudia Liebrand hat die in Der Sandmann vorzufindende "transzendetalpoetische[n] Reflexion“ hervorgehoben, bei der die Erzählinstanz mehr „weiß“ als seine Figuren (Liebrand 1996: 104-107).

5 So wird beispielsweise vom Serapionsbruder Theodor das „serapiontische Prinzip“ auf Leibniz“ Kettenoder Leitermetaphorik bezogen: „Ich meine, daß die Basis der Himmelsleiter, auf der man hinaufsteigen will in höhere Regionen, befestigt sein müsse im Leben, so daß jeder nachzusteigen vermag. Befindet er sich dann immer höher und höher hinaufgeklettert, in einem fantastischen Zauberreich, so wird er glauben, dies Reich gehöre auch noch in sein Leben hinein, und sei eigentlich der wunderbar herrlichste Teil desselben“" (IV: 721). 
auch verkürzt formulieren: Die Monade braucht bei Kant keinen „zureichenden Grund“ mehr, weil sie nun „Land“ okkupiert. Schon zu Kants Lebzeiten wurde sein Raumkonzept als Okkupationsprojekt eines ausgedehnten, dreidimensionalen Raumes von Salomon Maimon kritisiert. Für Maimon gibt es keine Unterscheidung zwischen Realopposition und logischer Opposition. Der stark von Leibniz beeinflusste Maimon kritisierte Kant, die Kausalitätskategorie „,on ihrem Gebrauche bei wirklichen Gegenständen abstrahiert, und in die Logik übertragen" (Maimon 2004: 44) zu haben. Im Rahmen seiner Entgegensetzung weist er das von Newton entwickelte Kräfteparallelogramm, welches Kant für seine Übertragung des Kausalitätsbegriffs aus der Kinematik auf die Logik benutzte, als empirisches Gesetz aus. Er kritisiert an diesem Parallelogramm und dessen vorausgesetztem Konzept der Realopposition, dass es als „mechanische Konstruktion“, die sich auf Kräfte und materielle Körper bezieht, nicht die Unabhängigkeit der Komponenten der zusammengesetzten Bewegung zu garantieren vermag (Freudenthal 2001: 18-19). J. G. Fichte sowie J. W. Goethe waren mit der skeptischen Kritik Maimons an Kant vertraut. So berichtet David Veits beispielsweise in einem Brief an Rahel Levin, dass Goethe ihn immer wieder nach Maimon gefragt habe.

Genau aus diesem Brief als eine der Notizen, die er sich über seine Lektüre des Buches Goethes Gespräche machte, exzerpiert Kafka in der Nacht vom 11. auf den 12.3.1912 (T: 403 u. T/Komm: 100, 110-113). ${ }^{6}$ Es kann davon ausgegangen werden, dass die Schriften Maimons unter Kafka und seinen Freunden bekannt waren. So empfiehlt er noch 1917 die von Karl Philipp Moritz herausgegebene Autobiographie Maimons seinem Freund Felix Weltsch zur Lektüre. Er beschreibt dabei Maimon als einen „zwischen Ost- und Westjudentum gespenstisch hinlaufenden Menschen“" (Kafka B 1914-1917: 371). 1932 verfasst Kafkas Schulfreund Samuel Hugo Bergmann in Jerusalem zudem eine Abhandlung über Salomon Maimon in hebräischer Sprache. Nicht nur in Bezug zu Kafkas jüdischer Sozialisation, sondern auch in Hinblick auf sein Schreibverfahren wird folgendes Er-Fragment immer wieder gelesen. Es vermag Kafkas Maimon-Lektüre ebenso zu veranschaulichen wie dessen Kritik am kantischen Kräfteparallelogramm:

Er hat zwei Gegner, der Erste bedrängt ihn von rückwärts vom Ursprung her, der Zweite verwehrt ihm den Weg nach vorne. Er kämpft mit beiden. Eigentlich unterstützt ihn der Erste im Kampf mit dem Zweiten, denn er will ihn nach vorne drängen und ebenso unterstützt ihn der Zweite im Kampf mit dem Ersten, denn er treibt ihn doch zurück. So ist es aber nur teoretisch, denn es sind ja nicht nur die 2 Gegner da, sondern auch noch er selbst und wer kennt eigentlich seine Absichten? Immerhin ist es sein Traum, dass er einmal in einem unbewachten Augenblick - dazu gehörte allerdings eine Nacht so finster wie noch keine war - aus der Kampflinie ausspringt und sich als ein Kampferfahrener zum Richter über die nun mit einander kämpfenden Gegner erhoben wird. (T: 851-852 u. T/App.: 398; Rechtschreibung im Original)

6 Kafkas Texte werden im Folgenden mit den Siglen B1914-1917 (=Briefe 1914-1917), DL/App (= Drucke zu Lebzeiten. Apparatband), T (=Tagebücher), T/Komm (= Tagebücher. Kommentarband), T/ App (= Tagebücher. Apparatband), NSFII (=Nachgelassene Schriften und Fragmente II) nach folgender Ausgabe zitiert: Franz Kafka: Schriften, Tagebücher, Briefe. Kritische Ausgabe (1982-2013). Hg. von G. Neumann u. a. Frankfurt a. M.: Fischer. Zudem wird der Text mit der Sigle OxOk4 (=Oxforder Oktavheft 4) nach der Faksimile-Ausgabe zitiert: Franz Kafka: Historisch-Kritische Ausgabe sämtlicher Handschriften, Drucke und Typoskripte (1995-2009). Hgg. von R. Reuß u. P. Staengle. Basel/Frankfurt a. M.: Stroemfeld. 
Die Zweiteilung des schreibenden Subjekts wird bei Kafka gerade nicht in eine aus einem Kräfteparallelogramm entspringende Diagonalkraft funktionalisierend ausgedehnt, ${ }^{7}$ sondern in seinem Widerspruch ausgehalten. Wie Maimon liegt Kafka eine Projektion von mechanischen Gesetzen auf die Logik fern, und wie jener geht er davon aus, dass ein „Widerstreit einen Widerspruch schon voraussetzt" (Maimon 1797: 45). Nicht durch den Widerstreit der zwei Hände und ihrer Kräfte kann daher das zitierte $E r$-Fragment als Doppelgängertext betrachtet werden, sondern durch seinen vorausgesetzten intelligiblen Widerspruch. Dieser Widerspruch ermöglicht einen Durchgang - nicht auf einer ausgedehnten Diagonalen, sondern als immer im Anfang bleibender, vor der Ausdehnung sich befindender Doppel-Gang ${ }^{8}$ zwischen einem „Er", der zu träumen vermag, und seinen Händen, die ihn gleichzeitig bekämpfen und unterstützen. Kafka realisiert hier Schrift einerseits als vom intelligiblen Menschen stammend, der sich durch sein Träumen ,auf dem geistigen Weg aus seiner Zelle“ (DL/App: 375) zu schleichen weiß. Andererseits wird hier Schreiben als vom körperlichen Menschen ausgehender Akt markiert, dem gleich einem Affen ,irgendwelche Schwebezustände der seligen Träume [...] nicht bereitet [sind]“ (DL/App: 375).

Da sich Kafka der Setzungsgewalt seines Schreibens, des von ihm in Sätzen intelligibel Gesetzten, bewusst ist, versucht er den Eingang dieses Gesetzten - den Raum vor dem Gesetz - als Doppel-Gang zu realisieren. Nicht nur können die gegenüber ihren medialen Stellvertretern auftretenden sinnlichen Doppelgänger dann vor dem Gesetz warten, an den aufgeklärten Selbstgerichtshof mit der Faust schlagen, in diesem Hof durch die klitzekleine Geste eines Fußtritts dessen moralischen Saustall bloßlegen, mit Erzähl-,und das heißt Gesetzesbeginn weiterschlafen oder eine anonyme Verleumdung in eine konkrete Rufschädigung umwandeln, sondern auch kräftig schimpfen. So regt sich im vierten Oxforder Oktavheft der Affe Rotpeter über einen über ihn schreibenden Journalisten auf, dem seiner Meinung nach ,jedes Fingerchen seiner schreibenden Hand einzeln weggeknallt werden“ (OxOkt4: 124) sollte. Kurz vorher findet man im gleichen Oktavheft Kafkas berühmtes Fragment Kampf der zwei Hände. In diesem bedauert ein Ich-Erzähler, dass er sein „Leben lang" die rechte vor der linken Hand bevorzugt habe:

\footnotetext{
7 Die Kantianerin Hannah Arendt bezichtigt Kafka, mit diesem Fragment den „alten Traum der abendländischen Metaphysik“ zu träumen, da sein „Er“ aus der Kausalkette von Tat und Beobachtung aussteigen möchte. Arendt zufolge darf das „Er“ nicht „einfach etwas Passives“ sein. Mit dem Verweis auf das Kräfteparallelogramm und Kants reflektierende Urteilskraft empfiehlt sie Kafkas „Er“ stattdessen den Gebrauch seiner „Diagonalkraft", die eine Bewegung in einem neutralen Zwischenraum der streitenden Kräfte ermöglichen würde (Arendt 1998: 203-211).

8 In ihrem Artikel Am Anfang war die Zwei. Doppelgängertexte und der doppelte Gang durch den Textanfang erklärt Erika Greber, dass Texte, die „das Anfangen“ performativ „inszenieren und praktizieren“, eine Rahmung zur Anfangspassage bilden, „die dadurch genau dieses sein kann:passage“ (Greber 2008: 195). Sie spricht davon, dass „literarische Texte hier etwas leisten können, was der diskursiven Reflexion des Anfangs entgeht“ (ebd.: 195), was gegen die Begrifflichkeit des „Ursprungs“ und damit gegen alle generischen Binäroppositionen gerichtet ist (ebd.: 197-199). Die Struktur von Doppelgängertexten weise wie ein Möbiusband „eine zirkuläre Struktur mit permanentem Ebenenwechsel“ (ebd.: 201) auf. Mit Verweis auf die bekannte Zeichnung von Maurits Cornelis Escher wird „die unlogische Struktur des Ebenenwechsels“ noch deutlicher als die „zwischen der gezeichneten und der zeichnenden Hand“ (ebd.: 202).
} 
Hätte doch die Linke einmal etwas gesagt, ich hätte nachgiebig, und rechtlich wie ich bin, gleich den Missbrauch eingestellt. Aber sie muckste nicht, hing an mir hinunter, und während etwa die Rechte auf der Gasse meinen Hut schwang, tastete die Linke ängstlich meinen Schenkel ab. Das war eine schlechte Vorbereitung zum Kampf, der jetzt vor sich geht. W[as]ie willst Du auf die Dauer linkes Handgelenk gegen dieses [sic] gewaltige Rechte Dich stemmen? Wie Deine mädchenhaften Finger in der Klemme der 5 andern behaupten? Das scheint mir kein Kampf mehr, sondern natürliches Ende der Linken. Schon ist sie in d[en]ie äussersten link[en]e [Winkel] Ecke des Tisches gedrängt, und an ihr regelmässig auf und nieder schwingend wie ein Maschinenkolben die Rechte. (OxOkt4: 104-107)

Der „Schreiber“ kann „die Linke“ nur retten durch einen „erlösenden Gedanken“: Er stellt fest, „dass es meine eigen [sic] Hände sind, die hier im Kampf stehn [sic] und dass ich sie mit einem leichten Ruck von einander wegziehen kann" (OxOkt4: 107-108). Die Aufhebung des Kampfes betrachtet der Ich-Erzähler hier als unehrlich, aber sie stellt für ihn die einzige Möglichkeit dar, das von ihm durch den Kampf der zwei Hände unterbrochene Lesen wiederaufzunehmen. Die Hände nehmen nach dem Kampf wieder ein Buch „vor“, welches sie vorher wegschieben mussten. Während das Lesen damit gewahrt wird, hat sich das Erzählen durch die unehrliche Aufhebung des Kampfes verändert. Wie wir schon wissen, ist nun statt dem „Er" der Affe Rotpeter Ich-Erzähler und auch Leser. Das Buch, welches seine Hände - oder sind es schon Pfoten? - zwischen dem verärgerten Zeitungslesen wieder „vor"nehmen, könnte auch Hoffmanns Lebensansichten des Katers Murr sein. Kafka selbst hat in jedem Fall kurz vor seinem Tod gerne im Kater Murr gelesen. Hatte schon der Hund Berganza der von ihm handelnden Erzählinstanz Tipps für den Druck seiner Berichte gegeben (1993: 136), so lässt Hoffmann in seinem Spätwerk einen Kater „seine“ Lebensansichten schreiben. Die Biographie des Johann Kreisler sei dabei nur versehentlich mit abgedruckt worden - als vom Kater zerrissene und auf der Rückseite benutzte Papierfetzen (2001: 11-14). Wie Sarah Kofmann hervorgehoben hat, bedeutet einen Krallen besitzenden „Kater schreiben [zu] lassen [...], die Schrift dem Leben selbst einzuschreiben, mit einer einzigen Geste die metaphysische Opposition zwischen Instinkt und Intelligenz durchzustreichen" (Kofmann 1985: 51).

In einer Art Doppelgängerschaft zu Hoffmanns Verschachtelung von diaristischen und prosaischen Textstrukturen ist es vielleicht kein oder gerade ein Zufall, dass Kafka sein letztes Tagebuchheft für Aufzeichnungen seiner Erzählung Forschungen eines Hundes verwendet hat und seine letzten Tagebuchaufzeichnungen - worauf Arno Dusini hinwies - „vom hinteren Ende her fortführte“, sodass sich der „letzte Tagebucheintrag Kafkas nicht am Ende, sondern inmitten des ,Zwölften Heftes' befindet“ (2005: 207). Mit dieser Mitte, die Kafka immer wieder performativ realisiert, entspricht er der von Maimon in verschiedensten Schriften wiederholten Feststellung, dass menschliche Erkenntnis „in der Mitte“ beginne (Maimon 2004: 190). Diese Mitte ist laut Kafka der fehlerhaften Tatsache geschuldet, als vermeintlich aufgeklärter „Bürger zweier Welten“, der sich durch seine physische Ausdehnung und staatsvertraglich geregelte Landnahme gleichzeitig frei und gesichert wähnt, an zwei voneinander getrennte Ketten gefesselt worden zu sein: „Will er nun auf die Erde drosselt ihn das Halsband des Himmels, will er in den Himmel jenes der Erde" (Kafka NSFII: 128). Oder ist hier jetzt gar - aber eben nicht augenscheinlich - von einem mit zwei Halsbändern versehenden Hund die Rede? 


\section{Literatur}

Arendt, Hannah (1998): Vom Leben des Geistes. Das Denken. Das Wollen. Hg. von Mary McCarthy. Aus dem Amerikanischen von Hermann Vetter. München/Berlin/Zürich: Piper.

Bachtin, Michail M. (2011): Zur Philosophie der Handlung. Aus dem Russischen von Dorothea Trottenberg. Hg., mit Anmerkungen und mit einem Vorwort von Sylvia Sasse. Berlin: Matthes \& Seitz.

Balke, Friedrich (2005): Wahnsinn der Anschauung. Kants Träume eines Geistersehers und ihr diskursives Apriori. In: Moritz Baßler, Bettina Gruber, Martina Wagner-Egelhaaf(Hgg.): Gespenster. Erscheinungen - Medien - Theorien. Würzburg: Königshausen \& Neumann, 297-313.

Dusini, Arno (2005): Tagebuch. Möglichkeiten einer Gattung. München: Fink.

Freudenthal, Gideon (2001): Maimon's Subversion of Kant's Critique of Pure Reason: There are no synthetic a priori judgments in physics. Berlin: Max-Planck-Institut für Wissenschaftsgeschichte.

Hoffmann, Ernst Theodor Amadeus (1985): Nachtstücke, Klein Zaches, Prinzessin Brambilla. Werke 1816-1820. Sämtliche Werke in 6 Bänden (1985-2004). Hgg. von H. Steinecke u. W. Segebrecht. Bd. 3. Frankfurt a. M.: Bibliothek deutscher Klassiker.

Hoffmann, Ernst Theodor Amadeus (1988): Die Elixiere des Teufels, Werke 1814-1816. Sämtliche Werke in 6 Bänden. Bd. 2/2. Frankfurt a. M.: Bibliothek deutscher Klassiker.

Hoffmann, Ernst Theodor Amadeus (1993): Fantasiestücke in Callot's Manier. Werke 1814. Sämtliche Werke in 6 Bänden. Bd. 2/1. Frankfurt a. M.: Bibliothek deutscher Klassiker.

Hoffmann, Ernst Theodor Amadeus (2001): Die Serapionsbrüder. Sämtliche Werke in 6 Bänden. Bd. 4. Frankfurt a. M.: Bibliothek deutscher Klassiker.

Kaulbach, Friedrich (1960): Die Metaphysik des Raumes bei Leibniz und Kant. Köln: Kölner Universitäts-Verlag.

Kafka, Franz (1990): Tagebücher u. Tagebücher. Apparatband u. Tagebücher. Kommentarband. Hgg. von H-G. Koch, M. Müller u. M. Pasley. In: Ders.: Schriften, Tagebücher, Briefe. Kritische Ausgabe (1982-2013). Hg. von G. Neumann u. a. Frankfurt a. M.: Fischer.

Kafka, Franz (1992): Nachgelassene Schriften und Fragmente II. Hg. von J. Schillemeit. In: Ders.: Schriften, Tagebücher, Briefe. Kritische Ausgabe (1982-2013). Hg. von G. Neumann u. a. Frankfurt a. M.: Fischer.

Kafka, Franz (1996): Drucke zu Lebzeiten. Apparatband. Hgg. von W. Kittler, H-G. Koch u. G. Neumann. In: Ders.: Schriften, Tagebücher, Briefe. Kritische Ausgabe (1982-2013). Hg. von G. Neumann u. a. Frankfurt a. M.: Fischer.

Kafka, Franz (2005): Briefe 1914-1917. Hg. von H-G. Koch. In: Ders.: Schriften, Tagebücher, Briefe. Kritische Ausgabe (1982-2013). Hg. von G. Neumann u. a. Frankfurt a. M.: Fischer.

Kafka, Franz (2008): Oxforder Oktavheft 4: In: Franz Kafka: Historisch-Kritische Ausgabe sämtlicher Handschriften, Drucke und Typoskripte (1995-2009). Hgg. von R. Reuß u. P. Staengle. Basel/ Frankfurt a. M.: Stroemfeld.

Kant, Immanuel (1766): Träume eines Geistersehers, erläutert durch Träume der Metaphysik. In: Ders.: Gesammelte Schriften. Hgg. von der Preussischen Akademie der Wissenschaften u. a. Berlin 1900ff. (AA), Bd. 2 (AAII), 315-373.

Kant, Immanuel (1798): Anthropologie in pragmatischer Hinsicht. In: Ders.: Gesammelte Schriften. Hgg. von der Preussischen Akademie der Wissenschaften u. a. Berlin 1900ff. Bd. 7 (AAVII), 117-333. 
Kohns, Oliver (2007): Die Verrücktheit des Sinns. Wahnsinn und Zeichen bei Kant, E. T. A. Hoffmann und Thomas Carlyle. Bielefeld: transcript.

Kofmann, Sarah (1985): Schreiben wie eine Katze. Zu E. T. A. Hoffmanns „Leben-Ansichten des Katers Murr". Hg. von Peter Engelmann. Graz/Wien: Böhlau.

Kittler, Friedrich (1994): Die Laterna magica der Literatur: Schillers und Hoffmanns Medienstrategien. In: Athenäum (4), 219-237.

Leibniz, Gottfried Wilhelm (1996): Hauptschriften zur Grundlegung der Philosophie. Teil 2. Hg. von Ernst Cassirer. Hamburg: Meiner.

Liebrand, Claudia (1996): Aporie des Kunstmythos. Die Texte E. T. A. Hoffmanns. Freiburg i. B.: Rombach.

Liebrand, Claudia (2009): Der Sandmann. In: Detlef Kremer (Hg.): E. T. A. Hoffmann. Leben-WerkWirkung. Berlin/New York: De Gruyter, 169-185.

Maimon, Salomon (1797): Kritische Untersuchungen über den menschlichen Geist oder das höhere Erkenntnis- und Willensvermögen. Hg. von Gerhard Fleischer dem Jüngeren. Leipzig.

Maimon, Salomon (2004): Versuch über die Transzendentalphilosophie. Eingeleitet und mit Anmerkungen sowie einer Beilage hg. von Florian Ehrensperger. Hamburg: Felix Meiner.

Steinecke, Günter (Hg.) (1997): E. T. A. Hoffmann. Stuttgart: Reclam.

Weissberg, Liliane (1990): Geistersprache. Philosophischer und literarischer Diskurs im späten achtzehnten Jahrhundert. Würzburg: Königshausen \& Neumann. 